Conference Proceedings Paper

\title{
Synthesis and Characterizations of 2D Platinum Diselenide
}

\author{
Irnik Dionisiev 1, Vera Marinova 1,*, Krastyo Buchkov ${ }^{1,2}$, Hristosko Dikov ${ }^{3}$, Ivalina Avramova ${ }^{4}$ \\ and Dimitre Dimitrov ${ }^{1,2}$ \\ 1 Institute of Optical Materials and Technologies, Bulgarian Academy of Sciences, Sofia, Bulgaria \\ 2 Institute of Solid State Physics, Bulgarian Academy of Sciences, Sofia, Bulgaria, Bulgaria \\ 3 Central Laboratory of Solar Energy and New Energy Sources, Bulgarian Academy of Sciences, Sofia, \\ Bulgaria \\ 4 Institute of General and Inorganic Chemistry Bulgarian Academy of Sciences, Sofia, Bulgaria \\ * Correspondence: vera_marinova@iomt.bas.bg
}

Received: 17 April 2020; Accepted: 7 May 2020; Published: 7 May 2020

\begin{abstract}
Platinum diselenide ( $\mathrm{PtSe}_{2}$ ), which belongs to the transition metals dichalcogenide (TMDCs) class of 2D materials, is characterized with a transition from semimetal to semiconductor with a thickness variation from bulk to monolayer and found versatile applications especially in sensors and mid-infrared detectors. In this study we report the large-scale synthesis of $\mathrm{PtSe}_{2}$ layers by thermally assisted selenization of pre-deposited platinum films in horizontal quartz-tube Chemical Vapour Deposition (CVD) reactor. Raman spectroscopy, X-ray diffraction (XRD) and Xray photoelectron spectroscopy (XPS) are used for characterization of the obtained 2D PtSez. It is observed that the Raman spectra of PtSez show strong dependence on the thickness (Pt deposition time). XPS analysis was applied to examine the chemical compositions in order to assess the quality of the synthesized $\mathrm{PtSe}_{2}$ films. All the studied properties reveal great potential to obtain continuous layers with controlled thickness and composition and further potential for integration in functional heterostructures for future nanoelectronic and optoelectronic devices.
\end{abstract}

Keywords: transition metal di-chalcogenides; $\mathrm{PtSe}_{2}$ films; thermally assisted conversion synthesis; XRD; XPS; Raman spectroscopy

\section{Introduction}

Noble TMDs (nTMDs) 2D materials, are displaying many fascinating properties including widely tunable bandgap, moderate carrier mobility, anisotropy, and high air stability [1-3].

The nTMDs and particularly the platinum diselenide PtSez show diverse chemical and physical properties which are intensively studied especially in a 2D form [4]. Their complex electronic structure with notable indirect band gap can be tuned by several factors as varying the number of layers [5], strain [6] or induced defects[7]. In monolayer form, the $\mathrm{PtSe}_{2}$ is semiconducting with a band gap in range: 1.2-1.8 eV [2]. It turns into a semimetal by increasing of number of layers. Moreover, the charge-carrier mobility of $\mathrm{PtSe}_{2}$ (values of $3000 \mathrm{~cm} / \mathrm{V} \cdot \mathrm{s}$ [4]) is among the highest in TMDCs and comparable to black phosphorus $\left(1000 \mathrm{~cm}^{2} / \mathrm{V} \cdot \mathrm{s}[8]\right)$ which make it promising candidate for high performance gas-sensing and photovoltaics, high-speed electronics and optoelectronics. $\mathrm{PtSe}_{2}$ films grown by thermally assisted conversion of platinum at a complementary metal-oxidesemiconductor (CMOS)-compatible temperature shows excellent promise for future applications, including integration into CMOS production lines [9].

Another notable advantage of $\mathrm{PtSe}_{2}$ is the environmental stability and appropriate synthesis requirements which are compatible with the conventional industrial technologies. The established synthesis approaches as Thermally Assisted Conversion (TAC) [4] and other methods as Chemical 
Vapor Deposition [10], Molecular Beam Epitaxy [11] shows fine options for $\mathrm{PtSe}_{2}$ nanostructure controllability and engineering for applications in opto-electronics, catalysis and sensors [12-14].

The present study systematizes the initial technological steps of TAC synthesis of $\mathrm{PtSe}_{2}$ and corresponding quality verification via structural and chemical characterization by XRD, Raman spectroscopy and XPS.

\section{Materials preparation and Methods.}

$\mathrm{PtSe}_{2}$ films were synthesized by two step TAC procedure [4]. The first (i) preparation stage facilitate a pre-deposition of Pt film using in a custom built magnetron sputtering system with 3 " diameter Pt target (99.99\% purity) by RF magnetron sputtering process. The sputtering gas was argon of $99.999 \%$ purity. The applied power was set to $300 \mathrm{~W}$ and the deposition pressure was $6 \times 10^{-1}$ torr. Substrate holder was rotating during the sputtering run. The thickness of the deposited Pt films was controlled by sputtering time. Pt thin films of varying thicknesses were deposited onto $\mathrm{SiO}_{2} / \mathrm{Si}$ and fused quartz substrate.

The second (ii) synthesis stage - a direct selenization of the pre-deposited films in a CVD reactor based on a dual zone tube furnace. The Se vapour (precursor powder) source and the Pt films on $\mathrm{Si} / \mathrm{SiO}_{2}$ are inserted as follows: in a low temperature zone $\left(\sim 220^{\circ} \mathrm{C}\right)$ and in the growth high temperature zone $\left(\sim 550{ }^{\circ} \mathrm{C}\right)$ respectively. The process is mediated via a carrier/reactive gas mixture flow of $95 \% \mathrm{Ar} / 5 \% \mathrm{H}_{2}$ for $2 \mathrm{~h}$ with consequent formation of another necessary gaseous precursor $\mathrm{H}_{2} \mathrm{Se}$ to enable the $\mathrm{PtSe}_{2}$ growth. A schematic of the CVD reactor is shown in Figure 1.

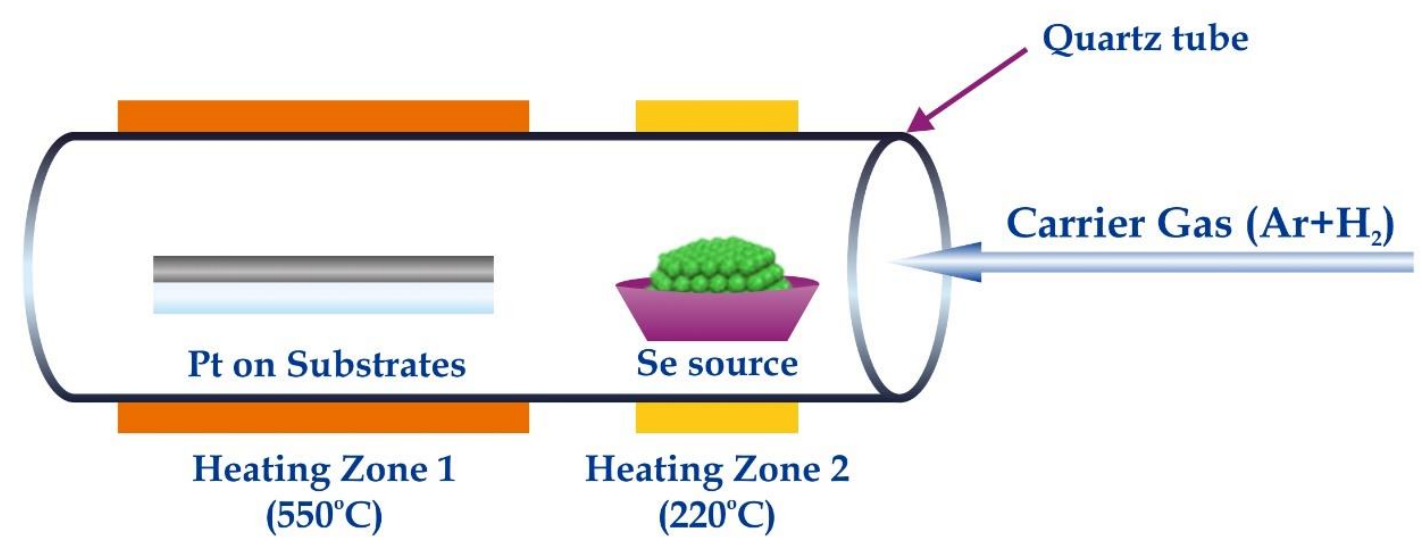

Figure 1. Schematic of CVD system with quartz tube reactor for synthesis of PtSe2 2D TMD material.

The focus of the study is the synthesis details, structural and chemical characterizations of PtSez layers obtained from a 30s Pt predeposition time. The X-ray diffractorgam patterns were collected using Bruker D8 Advance diffractometer with $\mathrm{Cu} K \alpha$ radiation and a LynxEye detector within the range of $5.3^{\circ}$ to $80^{\circ} 2 \theta$, with a constant step of $0.02^{\circ}$. The XPS analysis was performed using a Kratos AXIS Supra spectrometer with a non-monochromatic $\mathrm{Al} \mathrm{X-ray} \mathrm{source} \mathrm{under} \mathrm{vacuum}\left(<10^{-8} \mathrm{~Pa}\right)$ at 90 take-off angle. The acquired photoelectron spectra were additionally processed using background signal subtraction and fitting procedures routines of XPSPEAK41 software package. Raman spectroscopy measurements were carried out on an $80 \mathrm{~cm}$ Dilor XY-800, with triple monochromator allowing for multi-channel liquid nitrogen cooled CCD detection or single-channel PM detection. The experiments were performed at room temperature using $514.5 \mathrm{~nm}$ excitation line of a CW Ar+ laser.

\section{Results and Discussion}

The physical nature of 2D materials like TMDs and particularly $\mathrm{PtSe}_{2}$ is determined by the layered crystal structure (Figure 2) with van der Waals stacking and strong anisotropy of the properties. The XRD pattern identify the $\mathrm{PtSe}_{2}$ film crystal lattice with unit cell parameters determined to be $\mathrm{a}=3.728 \AA$ and $\mathrm{c}=5.06 \AA$ with hexagonal P3m1 [164] space group. The main (001) 
characteristic peak is detected approximately at $17.6^{\circ}$ degrees $2 \theta$. In the inset section an enlarged diffractogram sector (in the range $20-80^{\circ}$ ) is presented, showing the other peaks with diminished intensity as a consequence of the layered structure and dominant [001] orientation - implying a high crystalline quality and c-axis growth of the film plane. The peaks are assigned to (002), (003) and (004) crystallographic orientation and negligible traces $(<1 \%)$ of $\mathrm{Pt}$ and the substrate $\mathrm{SiO}_{2} / \mathrm{Si}(111)$ are also identified.

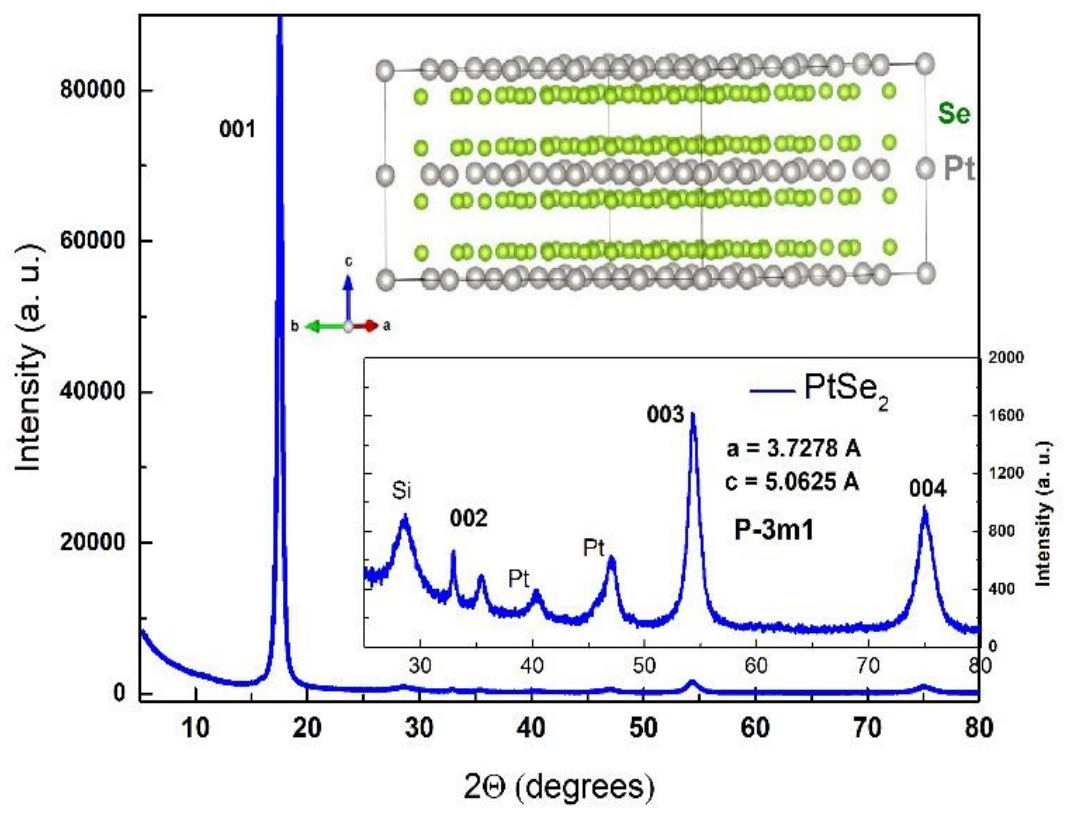

Figure 2. XRD spectra of $\mathrm{PtSe}_{2}$ film with a 30s deposition. The insets show an enlarged diffractogram sector with identified peaks and the layered nature of the crystal structure visualized by Vesta@ software [15].

The chemical composition and binding energies of the PtSez film was verified also by XPS. The spectrum (Figure 3) show the presence of both $\mathrm{Pt}$ and Se and the analysis data indicate that the $\mathrm{PtSe} 2$ phase is successfully formed. The Se $3 \mathrm{~d}$ peak is deconvoluted into two contributions: the main from $\mathrm{PtSe}_{2}$ appears at around $55 \mathrm{eV}$ (The spin-orbital splitting between Se $3 \mathrm{~d}_{5 / 2}$ and $\mathrm{Se} 3 \mathrm{~d}_{3 / 2}$ is $0.86 \mathrm{eV}$ ) and the minor with a binding energy around $59.5 \mathrm{eV}$ typical for Se oxide. There are also a traces for metallic state Pt in this range.

The $\mathrm{Pt} 4 \mathrm{f}$ is deconvoluted into two contributions, the one at $\sim 72.3 \mathrm{eV}$ is attributed to $\mathrm{PtO}_{\mathrm{x}} /$ hydroxide while the second one at $\sim 73.6 \mathrm{eV}$ was attributed to $\mathrm{PtSe} 2$. The spin-orbital splitting at $3.35 \mathrm{eV}$ was used for the fitting procedure. 


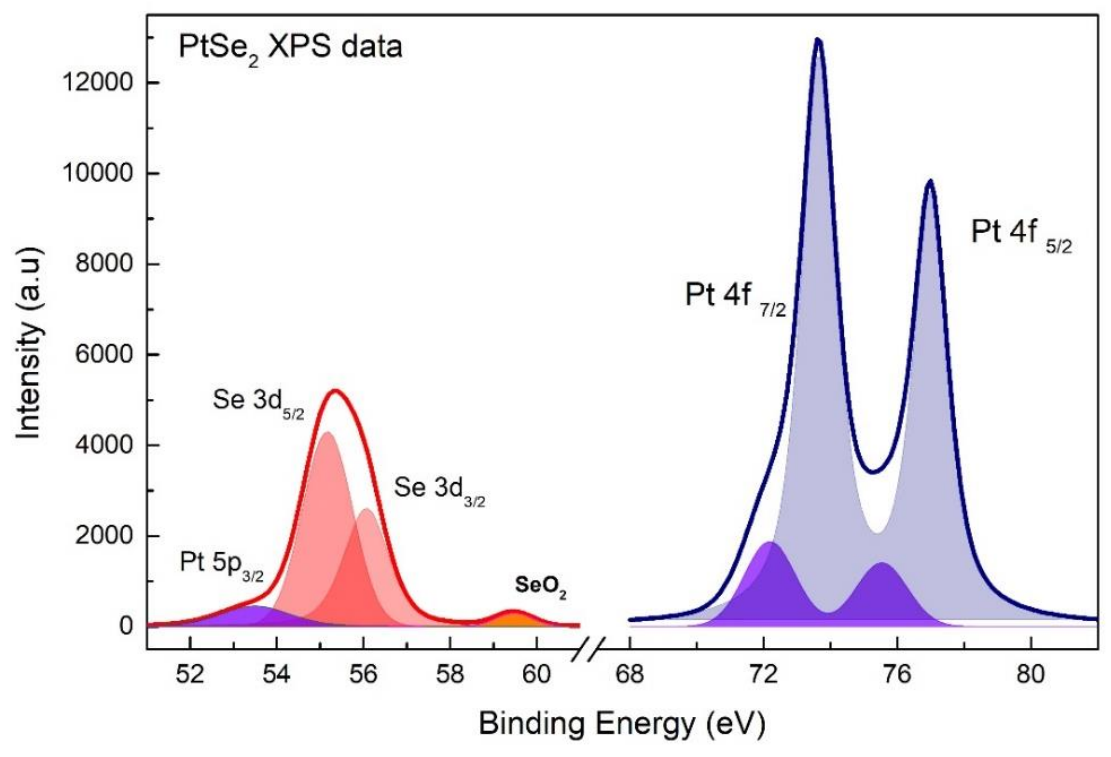

Figure 3. XPS spectral data for $\mathrm{PtSe}_{2}$

Raman spectroscopy is a powerful and nondestructive characterization technique which is widely used for 2D materials. The Raman spectra of TMDs are generally characterized by two main peaks corresponding to the in-plane and out-of-plane motions of atoms. The two Raman-active modes in the spectra of $\mathrm{PtSe}_{2}$ are labeled as $\mathrm{A}_{1 \mathrm{~g}}$ and $\mathrm{Eg}_{\mathrm{g}}$ [16]

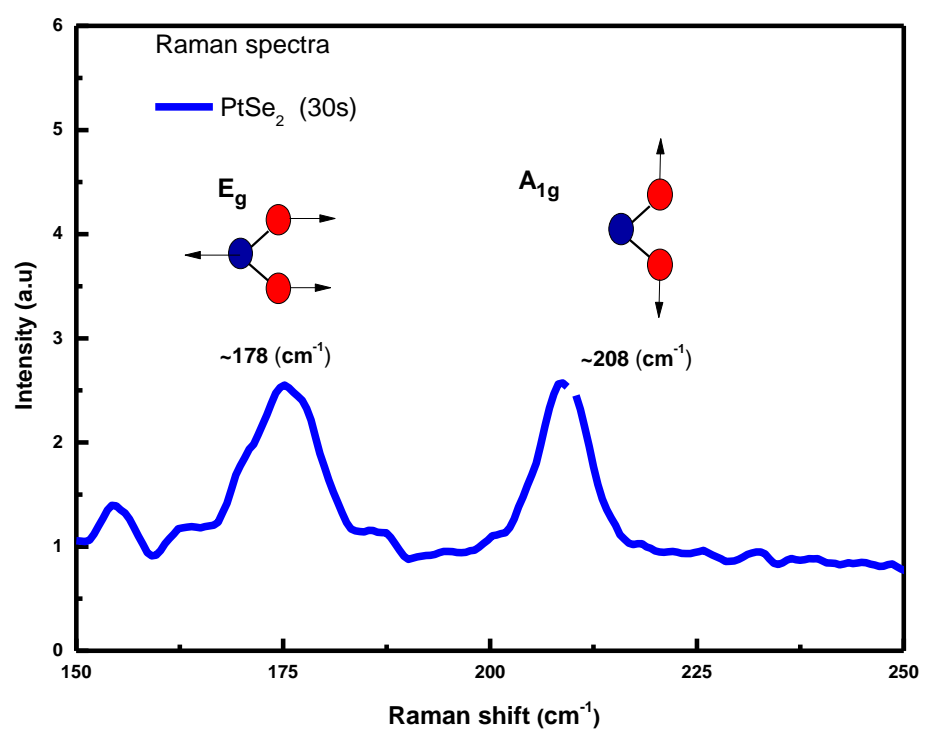

Figure 4. Raman spectra of PtSe2: Pt pre-sputtering time 30s on fused silica substrate at standard mode. A schematic of the vibrational modes in $\mathrm{PtSe} 2$ is also shown in the Figure.

A schematic of the vibrational modes in $\mathrm{PtSe}_{2}$ is shown in Figure 4, with arrows drawn as guides to show the origin of each mode on the phonon dispersion curve. These include the Eg mode, describing the in-plane vibration of selenium atoms in opposite directions within a single layer, and the Alg mode describing the out-of-plane vibration of selenium atoms. The characteristic Raman active $\mathrm{Eg}\left(178 \mathrm{~cm}^{-1}\right)$ and $\mathrm{A} 1 \mathrm{~g}\left(208 \mathrm{~cm}^{-1}\right)$ mode of TAC deposited PtSez confirm the composition and quality of the obtained samples. 


\section{Conclusions}

The layered 2D material $\mathrm{PtSe}_{2}$ was successfully synthesized by thermal assisted conversion process. The structural and chemical characterizations of the obtained $\mathrm{PtSe} 2$ confirm the composition and crystalline quality. The obtained results allow further directions for improvement of the preparation procedure (particular the deposition periods) to facilitate the nanostructure synthesis approach towards the 2D PtSe2 applications

Author Contributions: This article was coordinated by V.M., all authors giving input to the written text. In particular, V.M., D.D. and K.B. wrote most of the Introduction and Materials and Method, K.B. interpreted XRD analyses, I.A. performed and interpreted XPS analyses. D.D., H.D., and V.M. synthesized the PtSez samples. Raman measurements and interpretation of the data were done by D.D. with contribution from K.B. I.D. contributed to visualization and data acquisition. All authors have read and agreed to the published version of the manuscript.

Acknowledgments and Funding: This work is supported by the Bulgarian Ministry of Education and Science under the National Research Programme "Young scientists and postdoctoral students" approved by DCM\# 577/17.08.2018 and by Bulgarian Science Fund under the project КП-06-ДКОСТ/1.

Conflicts of Interest: The authors declare no conflict of interest

\section{References}

1. Wang, Z.; Li, Q.; Besenbacher, F.; Dong, M. Facile Synthesis of Single Crystal PtSe2Nanosheets for Nanoscale Electronics. Adv. Mater. 2016, 28, 10224-10229, doi:10.1002/adma.201602889.

2. Zhao, Y.; Qiao, J.; Yu, Z.; Yu, P.; Xu, K.; Lau, S.P.; Zhou, W.; Liu, Z.; Wang, X.; Ji, W.; et al. High-ElectronMobility and Air-STable 2D Layered PtSe2 FETs. Adv. Mater. 2017, 29, 1604230, doi:10.1002/adma.201604230.

3. Pi, L.; Li, L.; Liu, K.; Zhang, Q.; Li, H.; Zhai, T. Recent Progress on 2D Noble-Transition-Metal Dichalcogenides. Adv. Funct. Mater. 2019, 29, 1904932, doi:10.1002/adfm.201904932.

4. Wang, Y.; Li, L.; Yao, W.; Song, S.; Sun, J.T.; Pan, J.; Ren, X.; Li, C.; Okunishi, E.; Wang, Y.-Q.; et al. Monolayer PtSe 2 , a New Semiconducting Transition-Metal-Dichalcogenide, Epitaxially Grown by Direct Selenization of Pt. Nano Lett. 2015, 15, 4013-4018, doi:10.1021/acs.nanolett.5b00964.

5. Villaos, R.A.B.; Crisostomo, C.P.; Huang, Z.-Q.; Huang, S.-M.; Padama, A.A.B.; Albao, M.A.; Lin, H.; Chuang, F.-C. Thickness dependent electronic properties of Pt dichalcogenides. npj 2D Mater. Appl. 2019, 3, 2, doi:10.1038/s41699-018-0085-z.

6. Li, P.; Li, L.; Zeng, X.C. Tuning the electronic properties of monolayer and bilayer PtSe 2 via strain engineering. J. Mater. Chem. C 2016, 4, 3106-3112, doi:10.1039/C6TC00130K.

7. Absor, M.A.U.; Santoso, I.; Harsojo; Abraha, K.; Ishii, F.; Saito, M. Defect-induced large spin-orbit splitting in monolayer PtSe 2. Phys. Rev. B 2017, 96, 115128, doi:10.1103/PhysRevB.96.115128.

8. Li, L.; Yu, Y.; Ye, G.J.; Ge, Q.; Ou, X.; Wu, H.; Feng, D.; Chen, X.H.; Zhang, Y. Black phosphorus field-effect transistors. Nat. Nanotechnol. 2014, 9, 372-377, doi:10.1038/nnano.2014.35.

9. Wagner, S.; Yim, C.; McEvoy, N.; Kataria, S.; Yokaribas, V.; Kuc, A.; Pindl, S.; Fritzen, C.-P.; Heine, T.; Duesberg, G.S.; et al. Highly Sensitive Electromechanical Piezoresistive Pressure Sensors Based on LargeArea Layered PtSe 2 Films. Nano Lett. 2018, 18, 3738-3745, doi:10.1021/acs.nanolett.8b00928.

10. Yim, C.; Lee, K.; McEvoy, N.; O’Brien, M.; Riazimehr, S.; Berner, N.C.; Cullen, C.P.; Kotakoski, J.; Meyer, J.C.; Lemme, M.C.; et al. High-Performance Hybrid Electronic Devices from Layered PtSe 2 Films Grown at Low Temperature. ACS Nano 2016, 10, 9550-9558, doi:10.1021/acsnano.6b04898.

11. Yan, M.; Wang, E.; Zhou, X.; Zhang, G.; Zhang, H.; Zhang, K.; Yao, W.; Lu, N.; Yang, S.; Wu, S.; et al. High quality atomically thin PtSe 2 films grown by molecular beam epitaxy. 2D Mater. 2017, 4, 045015, doi:10.1088/2053-1583/aa8919.

12. Al Mutairi, A.; Yin, D.; Yoon, Y. PtSe 2 Field-Effect Transistors: New Opportunities for Electronic Devices. IEEE Electron Device Lett. 2018, 39, 151-154, doi:10.1109/LED.2017.2773599.

13. Sajjad, M.; Montes, E.; Singh, N.; Schwingenschlögl, U. Superior Gas Sensing Properties of Monolayer PtSe 2. Adv. Mater. Interfaces 2017, 4, 1600911, doi:10.1002/admi.201600911. 
14. Yim, C.; McEvoy, N.; Riazimehr, S.; Schneider, D.S.; Gity, F.; Monaghan, S.; Hurley, P.K.; Lemme, M.C.; Duesberg, G.S. Wide Spectral Photoresponse of Layered Platinum Diselenide-Based Photodiodes. Nano Lett. 2018, 18, 1794-1800, doi:10.1021/acs.nanolett.7b05000.

15. Momma, K.; Izumi, F.; IUCr; K., H.; H., N.; J., C.S.; D., S.M.; J., P.C.; J., H.P.; J., P.M.; et al. VESTA 3 for threedimensional visualization of crystal, volumetric and morphology data. J. Appl. Crystallogr. 2011, 44, 12721276, doi:10.1107/S0021889811038970.

16. O’Brien, M.; McEvoy, N.; Motta, C.; Zheng, J.-Y.; Berner, N.C.; Kotakoski, J.; Elibol, K.; Pennycook, T.J.; Meyer, J.C.; Yim, C.; et al. Raman characterization of platinum diselenide thin films. 2D Mater. 2016, 3, 021004, doi:10.1088/2053-1583/3/2/021004.

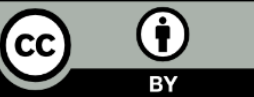

(C) 2020 by the authors. Submitted for possible open access publication under the terms and conditions of the Creative Commons Attribution (CC BY) license (http://creativecommons.org/licenses/by/4.0/). 